\title{
Experiences with the Telovelar Approach to Fourth Ventricular Tumors in Children
}

\author{
S. Noman Zaheer Martin Wood \\ Royal Children's Hospital, Brisbane, Qld., Australia
}

\section{Key Words}

Telovelar approach • Fourth ventricular tumor, children

\begin{abstract}
Background: Fourth ventricular tumors are amongst the most common tumors in the pediatric population. Traditionally, these tumors are approached through the cerebellar vermis, but the telovelar approach is now becoming widespread. Posterior fossa syndrome/cerebellar mutism is a complication of surgery for fourth ventricular tumors whose precise cause remains elusive, but may be related to the surgical approach or injury to adjacent cerebellar structures. We present a small series of fourth ventricular tumors and our initial experience in using the telovelar approach for this surgery. Methods: Twenty patients with fourth ventricular tumors were operated on using the microsurgical telovelar approach, sparing the cerebellar vermis. Data were collected prospectively for all patients with respect to the degree of resection achieved, complications and the incidence of posterior fossa syndrome. Results: A complete resection was achieved on postoperative MRI in $70 \%$ of the patients. Residual disease $<1.5 \mathrm{~cm}^{3}$ remained in $15 \%$ and a further $15 \%$ had subtotal resection with $>1.5 \mathrm{~cm}^{3}$ of residual disease. Thirty percent had evidence of posterior fossa syndrome in the postoperative period, of which $84 \%$ had resolved at the last follow-up. Thirty percent of the patients developed symptomatic hydrocephalus requiring shunting. There were no new neurological deficits and no procedure-related deaths. Conclusion: The telovelar approach provides excel-
\end{abstract}

lent access to tumors of the fourth ventricle with sparing of the vermis in children. The high incidence of cerebellar mutism in our patients confirms that this phenomenon is unlikely to be related to vermian injury and further study is recommended.

Copyright $\odot 2011$ S. Karger AG, Basel

Access to the fourth ventricle in the midline is required for the surgical treatment of many common pediatric brain tumors. Traditional approaches have involved a large suboccipital craniotomy followed by splitting of the cerebellar vermis in the midline or a transcerebellar approach to gain access to the ventricle. In recent years, there has been significant focus on the potential adverse effects of surgical damage to the midline cerebellar structures, with some evidence for increased incidence of postoperative cerebellar mutism and neurocognitive deficits [1]. Since 2007, the senior author (M.W.) has preferentially used the vermis-sparing, minimally traumatic telovelar approach for midline posterior fossa tumors in children, and the initial experiences and results of this approach are presented.

\section{Clinical Methods}

From June 2007, all patients presenting with fourth ventricular tumors were operated upon via the midline suboccipital telovelar approach. Data relating to patient demographics, histology, degree of resection, complications and the incidence of pos-

\section{KARGER}

Fax +4161306 1234 E-Mail karger@karger.ch www.karger.com

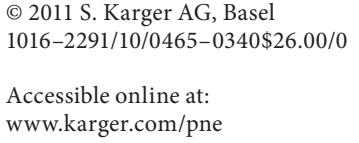

Dr. S. Noman Zaheer

Unit 18, 37 Playfield Street

Brisbane, QLD 4032 (Australia)

Tel. +61 733590053

E-Mail drnomanzaheer@yahoo.com 
terior fossa syndrome (postoperative 'cerebellar mutism' syndrome) were recorded prospectively.

All patients underwent MRI scanning within $72 \mathrm{~h}$ postoperatively to determine the degree of resection obtained. These images were reviewed by a consultant pediatric neuroradiologist, the treating surgeon and the treating oncologist. A consensus decision was reached about the degree of resection.

'Posterior fossa syndrome' was defined as the presence of partial or complete loss of spontaneous speech, either in isolation or together with other features such as decreased spontaneous movements, hypersomnia, listlessness or visual inattention, in the absence of any reversible causative factor on the postoperative imaging such as hematoma or hydrocephalus.

\section{Surgical Technique}

The microsurgical anatomy of the telovelar access to the fourth ventricle has been well described previously by Mussi and Rhoton [2]. The surgical technique employed in this series is described as follows.

All procedures were performed with the patient in the prone position with the head flexed at the craniocervical junction. $\mathrm{Pa}-$ tients aged $<2$ years were not placed in rigid skull fixation; instead, the head was placed on a horseshoe rest with tape placed around the vertex and attached to the headrest to maintain stability of positioning. After making a midline occipitocervical incision extending from the inion to the level of the spinous process of $\mathrm{C} 2$, the occipital bone and posterior arch of $\mathrm{C} 1$ were exposed. A small midline suboccipital craniotomy was cut, exposing only the cerebellar tonsils and inferior vermis. The posterior arch of $\mathrm{C} 1$ was removed if the tonsils were seen descend to this level or below on the preoperative imaging. It was not necessary to expose the bulk of the cerebellar hemispheres. A U-shaped dural opening was made with the base of the flap sited superiorly. Under the operating microscope, the cerebellar tonsil on the side of the greatest tumor bulk was gently elevated to reveal the foramen of Magendie, the tela choroidea and the inferior medullary velum. The inferior aspect of a large tumor was often evident protruding through the foramen of Magendie, with the tela choroidea and inferior medullary velum stretched thinly over its surface. In fact, there was less dissection of these structures required in cases where the tumor was large, due to the distension at the foramen of Magendie produced by the tumor. The posterior inferior cerebellar artery was also usually visualized at this point and care taken to protect it on each side. Whilst the ipsilateral cerebellar tonsil was elevated over a cotton patty to expose the cerebellomedullary fissure, the inferior medullary velum was sharply dissected to gain access to the tumor and (after its removal) the main portion of the fourth ventricle; if necessary, the tela choroidea was also divided to access the lateral recess of the ventricle. The vermis was not dissected nor was any incision made in the cerebellar hemisphere. The tumor was then dissected from surrounding cerebellar and brainstem structures using microsurgical techniques, with the bulk of the resection performed with the ultrasonic aspirator. At the conclusion of the resection of larger tumors, the roof of the ventricle was inspected with the use of a 30-degree rigid endoscope, passed through the foramen of Magendie, to determine whether all tumor tissue had been removed. The suboccipital bone flap was replaced in all patients. Postoperative external ventricular drainage was not routinely used.

Telovelar Approach and Fourth

Ventricular Tumors

\section{Results}

\section{Patient Details}

Twenty patients presented with fourth ventricular tumors between June 2007 and December 2009. There were 11 boys and 9 girls, with ages ranging between 1 and 17 years (mean $=8$ years). Preresection treatment of symptomatic hydrocephalus was required in 6 patients; this was achieved by endoscopic third ventriculostomy in 3 patients and placement of an external ventricular drain in a further 3 .

\section{Histology}

The tumor histology was medulloblastoma in 14 patients, pilocytic astrocytoma in 3, ependymoma in 2 and a high-grade astrocytoma in 1.

\section{Degree of Resection}

A complete resection on postoperative MRI was obtained in 14 patients, with a small $\left(>1.5 \mathrm{~cm}^{3}\right)$ residual remaining in 3 ( 1 medulloblastoma, 1 pilocytic astrocytoma and 1 ependymoma). In the patient with the ependymoma, the residual tumor was within the cerebellopontine angle and was completely resected at a second procedure via a lateral approach. In 1 medulloblastoma case the residual tumor was $>1.5 \mathrm{~cm}^{3}$. This patient was $<2$ years of age, had a very vascular tumor and suffered a large intraoperative blood loss requiring massive transfusion. Further treatment with chemotherapy was administered and a subsequent surgical resection undertaken, with tumor clearance. One patient with a large cervicomedullary tumor had a significant residual tumor within the cervical spinal cord, and a further patient with a diffuse pontine glioma underwent a limited resection only to decompress a large cyst in the fourth ventricle causing brainstem dysfunction and hydrocephalus.

Six patients (30\%) developed symptoms in the postoperative period consistent with posterior fossa syndrome. In 4 patients this was mild, with a relative paucity of speech lasting only a few days. In the other 2 patients the deficits were more profound, with complete absence of voluntary speech, somnolence, hypotonia and decreased voluntary limb movements. One of these more severely affected patients (aged 18 months) had apparent visual loss bilaterally, without evidence of damage to any part of the visual pathway. Five patients had resolution of their symptoms within 1 month of onset, with the final patient experiencing ongoing deficits in speech 8 months postoperatively.

Pediatr Neurosurg 2010;46:340-343 


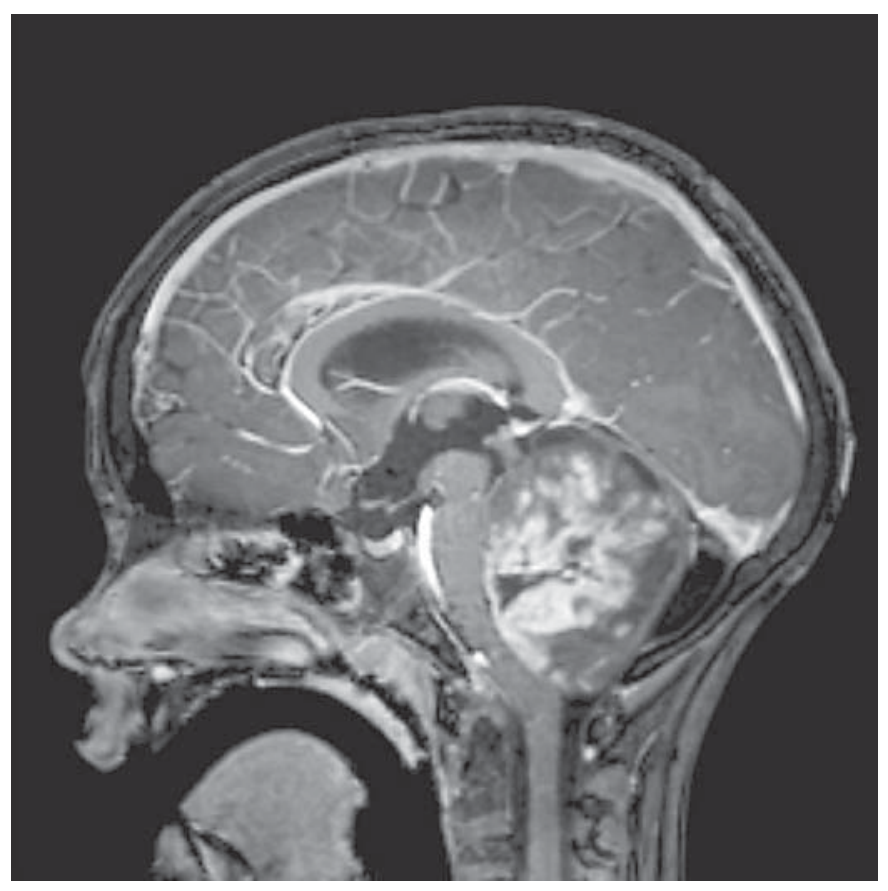

Fig. 1. Sagittal view (preoperative) of a large fourth ventricular pilocytic astrocytoma.

\section{Complications}

Six patients (30\%) developed postoperative communicating hydrocephalus requiring placement of a ventriculoperitoneal shunt. One of these shunts was subsequently removed, without recurrence of the hydrocephalus. In addition to these, 2 patients (10\%) developed a CSF leak from the surgical wound in the absence of hydrocephalus, 1 responding to suture placement and 1 requiring surgical debridement and reclosure of the wound.

There were no patients with new focal neurological deficit and no deaths related to surgery. Three patients died from early relapse of medulloblastoma following maximal treatment.

\section{Discussion}

The traditional approach to tumors within the fourth ventricle in children has involved incision of the cerebellar vermis after a midline suboccipital craniotomy or craniectomy. Whilst providing good access to the ventricle and facilitating removal of the tumor, this approach is not without its problems, and it has been postulated that vermian incisions correlate with a higher incidence of the 'cerebellar mutism' or 'posterior fossa syndrome' (both

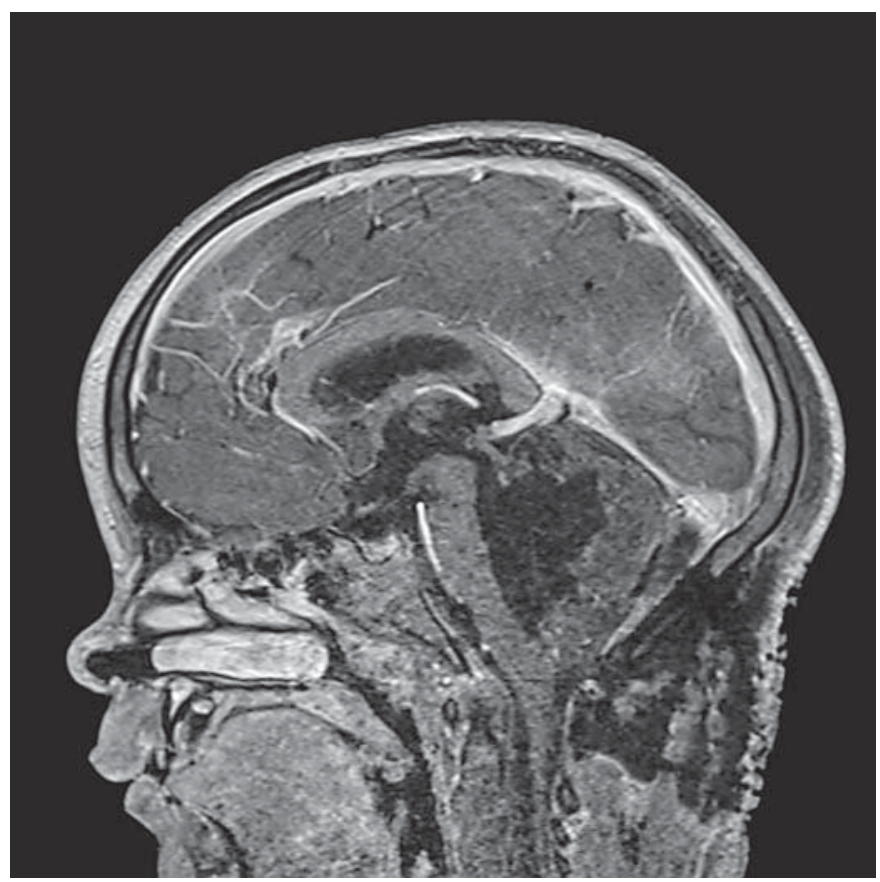

Fig. 2. Midline sagittal view (postoperative), demonstrating complete resection through the telovelar approach.

terms often being used interchangeably), together with longer-term neurocognitive sequelae [1]. Efforts have therefore been made to explore routes of access that avoid damage to the vermis or hemispheres of the cerebellum, in an attempt to minimize some of these postoperative deficits. The telovelar approach to the fourth ventricle has been previously well described and appears to provide a relatively minimally traumatic and vermian-sparing corridor into the space occupied by many posterior fossa tumors of childhood $[2,3]$.

This study is a small prospective series of children with midline fourth ventricular tumors, all of whom were operated upon via a midline, suboccipital, telovelar approach in the prone position. Thirteen of 20 patients (65\%) had complete resection of their tumors via this approach, demonstrating that whilst the telovelar access corridor may be narrower than that provided by a generous vermian-splitting approach, it is possible to completely resect even large tumors without difficulty (fig. 1). In fact, the larger the tumor preoperatively, the more stretched and thinned the inferior medullary velum and tela choroidea tends to be, which facilitates easier and wider access to the tumor within the ventricle (fig. 2, 3).

Our incidence of posterior fossa syndrome symptoms in this series was $30 \%$. This would seem to be contradic- 
Fig. 3. Artist's depiction of the telovelar access to the fourth ventricle via a midline suboccipital approach. Incision 1 opens access to the body and roof of the fourth ventricle. Incision 2 provides access to the lateral recess of the fourth ventricle. FM = Foramen of Magendie; Uv = uvula; IMV = inferior medullary velum; TC = tela choroidea; Floc $=$ flocculus.

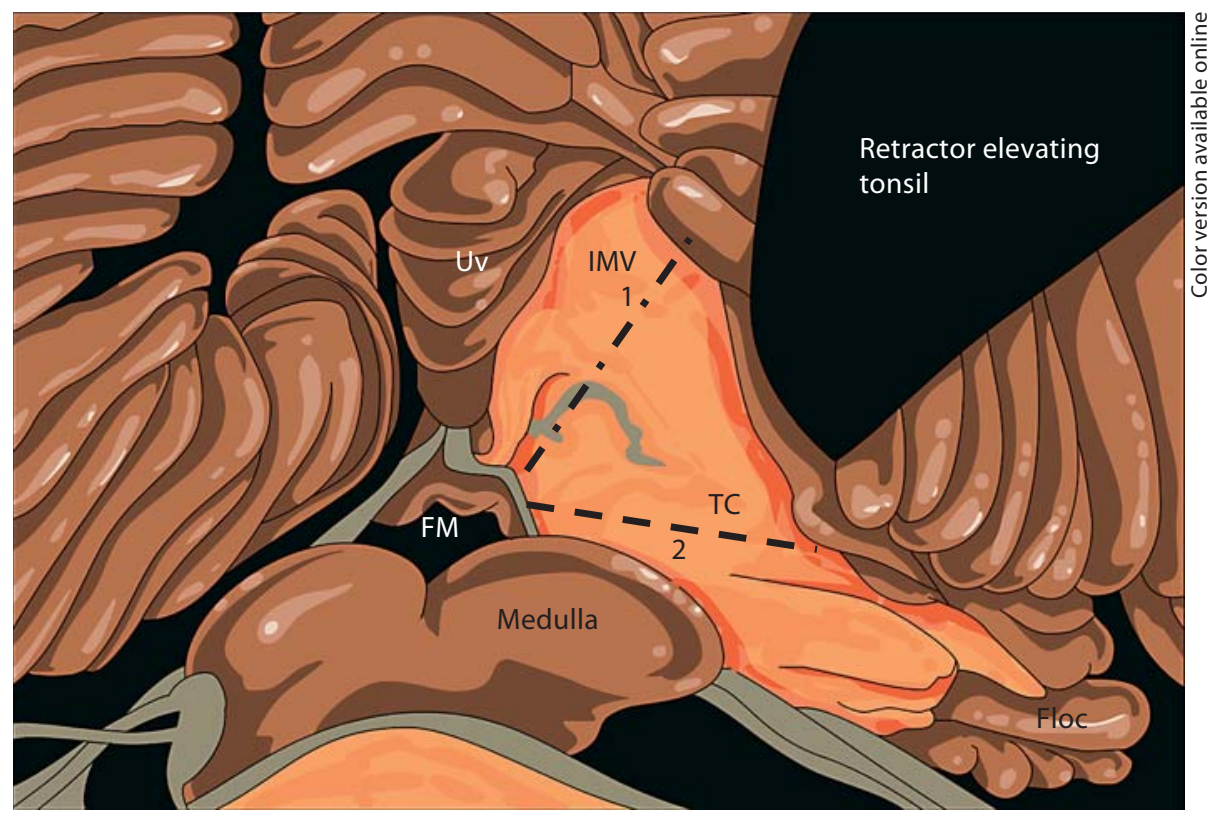

tory to the notion that dissection of the cerebellar vermis contributes significantly to the symptoms seen in posterior fossa syndrome postoperatively, as the telovelar approach obviates the need for any dissection of the vermis. Previous studies of the incidence of posterior fossa syndrome put its occurrence at a rate of between 8 and 24\% [4-6]. It is thought that the dentate nuclei and their connections through the brainstem to the thalamus have a role in speech function and that it is damage to this pathway that results in the production of the mutism syndrome. The higher incidence of this syndrome in our series may reflect the greater sensitivity of a prospective series for complications such as this, but it may also be due to the small size of the series and a chance occurrence. An additional factor could be that it is symptomatic of a relatively aggressive surgical approach with pur- suit of complete resection in all patients if possible. This may lead to a higher incidence of injury or postoperative edema within the dentate nuclei, particularly in patients with medulloblastoma in whom the tumor usually arises from the vermis, invading the brain close to the position of the dentate nuclei. However, given the risk stratification of medulloblastoma on modern treatment protocols according to volume of residual tumor, it is generally accepted that complete resection should be the goal of surgery, so our patient series is probably fairly typical of the type and extent of surgery within most pediatric centers. The high incidence of posterior fossa syndrome in our study though suggests that it may be more common than is generally held to be the case, and larger prospective studies of its occurrence are warranted if we are to better understand this interesting phenomenon.

\section{References}

1 Grill J, Viguier D, Kieffer V, et al: Critical risk factors for intellectual impairment in children with posterior fossa tumors: the role of cerebellar damage. J Neurosurg 2004; 101(suppl 2):152-158

2 Mussi A, Rhoton A: Telovelar approach to the fourth ventricle: microsurgical anatomy. J Neuosurg 2000;92:812-823.

Telovelar Approach and Fourth Ventricular Tumors
3 Deshmukh V, Figueiredo E, Deshmukh P, et al: Quantification and comparison of telovelar and transvermian approaches to the fourth ventricle. Neurosurgery 2006;58 (suppl 2):ONS 202-206.

4 Robertson P, Muraszko K, Holmes E, et al: Incidence and severity of postoperative cerebellar mutism syndrome in children with medulloblastoma: a prospective study by the Children's Oncology Group. J Neurosurg Pediatrics 2006; 105:444-451.
5 Doxey D, Bruce D, Sklar F, Swift D, Shapiro $\mathrm{K}$ : Posterior fossa syndrome: identifiable risk factors and irreversible complications. Pediatr Neurosurg 1999;31:131-136.

6 Pollack I, Polinko P, Albright AL, Towbin R, Fitz C: Mutism and pseudobulbar symptoms after resection of posterior fossa tumors in children: incidence and pathophysiology. Neurosurgery 1996;38:1066. 